\title{
ANÁLISIS DE LA COMPLEJIDAD SINTÁCTICA DE TEXTOS PRODUCIDOS POR ALUMNOS CON BAJA VISIÓN, CON CEGUERA Y CON VISIÓN NORMAL
}

\author{
Antonio Rodríguez Fuentes \\ Universidad de Granada, España
}

\section{INTRODUCCIÓN}

El presente artículo contiene resultados parciales de una investigación más amplia que versa sobre la comunicación escrita de alumnos con baja visión e invidentes, realizada durante un lustro con objeto de la formalización de la Tesis Doctoral, ya defendida (Rodríguez, 2002). Ha sido financiada por la Junta de Andalucía, mediante la concesión de una beca de FPDI (Formación de Personal Docente e Investigador) y se ha desarrollado en el seno del Grupo ED.INVEST de la Universidad de Granada. A su vez, se inserta en un macroproyecto cuyo objeto es el análisis de la competencia y las dificultades en la expresión escrita de alumnos con n.e.e., cuya relevancia ha sido reconocida por el Ministerio de Ciencia y Tecnología como proyecto de Investigación + Desarrollo, dotándolo de la financiación correspondiente.

La investigación realizada muestra interés por una perspectiva bifronte: de una parte, la lingüística y también la psicolingüística y, de otra, la didáctica. Sin menoscabo de la primera, es la última la que orienta este trabajo; si bien, la preocupación por ella pasa por el obligado análisis previo de aquélla. En definitiva, se trata de determinar pormenorizadamente el nivel de competencia sintáctica de alumnos con problemas severos de visión. La proyección u aportación didáctica de la investigación deriva, por tanto, de la fase diagnóstica de la misma y de sus propias conclusiones, que suponen un apoyo sustancial a la Didáctica de la Lengua y, más concretamente, al Proyecto Curricular en el área de Lengua. Todo ello en el caso de alumnos con baja visión e invidentes, donde la investigación referida se encuentra notablemente desfasada con respecto a alumnos sin deficiencias o incluso con otras deficiencias, como la auditiva o la mental.

\section{MARCO TEÓRICO DE LA INVESTIGACIÓN}

Tanto la adquisición como el dominio de la expresión escrita representan el culmen del aprendizaje lingüístico que comienza con el lenguaje oral y continúa con la lectura (Salvador, 1997). En efecto, el desarrollo de la composición escrita es complejo, como lo es también su didáctica. Desarrollo que se vertebra en diversas facetas, como la gráfica, gramática, semántica, sintaxis, etc. El sintáctico es el plano que interesa en esta ocasión, plano cuyo desarrollo resulta especialmente arduo. Esta competencia varía de unos países a otros, dadas las diferencias idiomáticas y de la didáctica de cada contexto. Obviamente, también existe una notable diversidad individual, dado la competencia lingüística de cada individuo. Ello advierte de la cautela que se ha de tener en cuanto a la generalización de los datos obtenidos en este proceso de investigación y en otros similares a éste.

Antes de aludir al estado de la investigación conviene traer a colación las distintas modalidades de investigación para los propósitos que se enumeran en el siguiente apartado. Tradicionalmente, se ha empleado el método experimental, método científico por excelencia. Aunque resulta viable la utilización de 
este método, la creciente fundamentación psicológica de la expresión lingüística unida a la emergencia de nuevos métodos de indagación o mejor la revalorización positiva de los mismos (cientificidad) han impulsado la aparición de investigaciones originales basadas en estudios de casos, investigaciones etnográficas, análisis de cuasi-producto, etc. La complementariedad de enfoques o perspectivas de análisis ha enriquecido, sin duda, el panorama explicativo de la composición escrita, en general, y sintáctica, en particular. En consecuencia, proliferan estudios sobre los procesos cognitivos de la expresión escrita así como sobre los productos de los mismos: los textos. No obstante, no son tan abundantes en nuestro panorama nacional, especialmente los dedicados a los procesos cognitivos, debido a la reciente consideración de los mismos (modelos cognitivos sobre la expresión escrita).

Tampoco menudean investigaciones cuya empresa es el estudio de la expresión escrita de alumnos con n.e.e. y, en concreto, la de alumnos invidentes y con baja visión. En nuestro contexto, esta línea de investigación resulta casi inédita (Rodríguez, 2003), pese a que sí existen obras sobre procesos similares al que nos ocupa, como es el estudio del lenguaje oral y de la lectura y sus disfunciones, aunque sin duda también resultan insuficientes (Rodríguez, 2005). En ambos casos, el lenguaje oral y la lectura en deficientes visuales, se advierten posibles desfases o particularidades e incluso dificultades y limitaciones que requieren nuevas investigaciones para ser identificadas definitivamente, especialmente en la lectura, dada su alta demanda visual. El hecho de que existan ciertos desfases, particularidades y limitaciones aconseja revisar también el desarrollo, las dificultades y las limitaciones potenciales que pudieran manifestar la expresión escrita. De otra parte, el análisis de la escritura tiene relevancia propia, dada la importancia de la propia actividad escritora. Los trabajos encontrados en nuestro panorama geográfico, impulsados por la ONCE y algunas Universidades, versan acerca de los procesos neurológicos, coordinación óculo-manual, motores y psicomotrices imbricados en la escritura, así como sobre los recursos tecnológicos al servicio de estos sujetos para posibilitar o coadyuvar su expresión por escrito. Se han puesto de relieve algunas dificultades, como la caligrafía, los errores gráficos y la presentación formal del texto que no propician la legibilidad del texto, y algunas limitaciones, como la lentitud escritora y la incomodidad de la tarea por los recursos requeridos (iluminación adicional, lentes ópticas y recursos tecnológicos).

Evidentemente, el análisis de la expresión escrita de alumnos con discapacidades visuales requiere ineludiblemente distinguir dos estados visuales por su alta incidencia en el hecho estudiado, pues cada uno de ellos demandará un sistema de escritura diferente: método en relieve Braille versus método de letra impresa en tinta tradicional. Se trata de los estados de ceguera e hipovisión, respectivamente. En efecto, obviamente el primer estado requiere del sistema Braille para su comunicación por escrito al contrario del segundo que, con las ayudas ópticas y no ópticas pertinentes y la implementación de programas de estimulación visuales, puede hacer uso del método de lectoescritura tradicional o bien adaptado (ampliado y/o resaltado). Aunque en determinados casos, es recomendable o necesario que el niño con escasa visión aprenda y use el sistema Braille, de forma complementaria al tradicional o alternativa. La dilucidación dilemática vendrá determinada por la evaluación oftalmológica y psicopedagógica que se ha de realizar con objeto de determinar las n.e.e. de estos alumnos, entre ellas la modalidad de lectoescritura. 


\section{OBJETIVOS Y METODOLOGÍA DE LA INVESTIGACIÓN}

El objetivo general de esta investigación es descubrir la complejidad sintáctica de los textos producidos por alumnos con baja visión e invidentes, como indicador, junto con otros como las disfunciones sintácticas, del grado de desarrollo sintáctico alcanzado por los sujetos de la investigación. Objetivos operativos derivados son los siguientes:

- Cuantificar la complejidad de los textos producidos en función al número de proposiciones en relación al total de oraciones

- Detectar la complejidad textual de acuerdo con la frecuencia de oraciones según su tipología: simples, yuxtapuestas, coordinadas y subordinadas en todos sus grados.

- Observar la complejidad oracional, de acuerdo con el número de oraciones por coordinación y yuxtaposición en función de las oraciones por subordinación

- Valorar la complejidad proposicional, de acuerdo con la cantidad de proposiciones coordinadas y yuxtapuestas en función del número de subordinadas.

Estos objetivos se consiguieron a través de los índices de complejidad utilizados, que se describen a continuación:

a) Índice de complejidad numérica, utilizado por algunos autores (Cfr. Simon, 1973; Salvador, 1984), que relaciona el número de proposiciones con el número de oraciones:

$$
\mathrm{Cp1}=\frac{\mathrm{n}^{\mathbf{0}} \text { de proposiciones X } 10}{\mathrm{n}^{\mathbf{0}} \text { de oraciones }}
$$

b) Índice ponderado de complejidad, diseñado por Salvador (1984), en el que se asigna un valor numérico a cada tipo de oración (Oración simple $(A)=1$, oración por yuxtaposición $(B)=2$, oración por coordinación $(C)=2$, oración por subordinación de primer grado $(D)=3$, oración por subordinación de segundo $(E)=4$ y oración por subordinación de tercer grado $(F)=5)$. La expresión matemática es la siguiente:

$$
\mathrm{Cp} 2=\frac{A+2 \cdot(B+C)+3 \cdot C+4 \cdot D+5 \cdot F}{n^{0} \text { de oraciones }}
$$

c) Índice de complejidad estructural de las oraciones, que determina un valor que expresa la relación entre las oraciones compuestas por coordinación y por yuxtaposición, de similar complejidad sintáctica, y las oraciones compuestas por subordinación, de mayor complejidad sintáctica. La fórmula matemática es la siguiente (Salvador, 1984): 


\section{Cp3 $=\frac{\text { Coordinación }+ \text { Yuxtaposición }}{\text { Subordinación }}$ \\ Subordinación}

d) Índice de complejidad estructural de las proposiciones de las oraciones compuestas, equivalente al anterior pero en el que las variables son las proposiciones, en lugar de las oraciones (Salvador, 1984):

$$
\text { Cp4 }=\underline{\text { Coordinadas }+ \text { Yuxtapuestas }}
$$

Subordinadas

En cuanto a la estrategia de investigación se ha empleado el estudio de casos, materializado en el análisis de textos narrativos de redacción libre (temática, amplitud y caracterización). Se le sugirió a los alumnos investigados que redactaran un texto narrativo, proporcionándoles una breve descripción o definición del mismo, a saber, que se trataba de relatar una historia sobre algún acontecimiento que le hubiera ocurrido, alguna aventura, alguna película o libro que hubiesen leído. Para ello se le entregaron dos hojas de papel pautado (formato A4) cuyas líneas y espacios interlineales resultaban apropiados para alumnos con baja visión, según las pautas sugeridas por algunos autores (Barraga, 1990; Rodríguez y Martínez, 2002). Con ello, se pretendía facilitar la grafía, la continuidad dentro del renglón y la separación adecuada entre ellos, con el doble objetivo de mejorar la grafía, optimizando la legibilidad del texto, a la vez que se conseguía que el alumno se concentrase en procesos más relevantes para esta investigación de mayor requerimiento o exigencia cognitiva que el diseño gráfico. De otra parte, los alumnos ciegos realizaron los textos en Braille, que fueron traducidos fielmente por profesoras especialistas integrantes del equipo de apoyo a la educación integrada de ciegos y deficientes visuales de Granada (EAICDV), colaboradoras de esta investigación, o bien fueron elaborados con el PC Hablado y posteriormente fueron almacenados y transferidos a un diskette estándar (1.44MB) e impresos mediante una impresora convencional. La ONCE y, concretamente, el EAICDV de Granada han proporcionado los medios necesarios para realizar la transcripción.

Existen diversas variantes para producir textos: dictados, copia y elaboración personal, cada una con unas intenciones determinadas. En congruencia con los objetivos planteados se optó por la última: la elaboración personal de un texto narrativo, con las siguientes características: a) tema libre, para que la producción sea lo más espontánea posible, a pesar de que algunos investigadores afirman que la variable independiente "tema libre / tema impuesto" ha resultado irrelevante en la producción realizada; b) contexto normalizado y familiar para el alumno, que ha sido generalmente el aula de apoyo del mismo centro en la que los profesores del EAICDV atienden al alumno, a excepción de aquellos que acuden asiduamente, con una periodicidad semanal o quinquenal, al centro de recursos del EAICDV, en cuyo caso, dicho centro ha sido el contexto de la investigación; c) no se ha impuesto ningún tipo de restricciones ni limitaciones, temporales ni espaciales, para la elaboración de su discurso; d) aunque la observación de la elaboración textual era imprescindible, se ha procurado que el alumno no se sintiese examinado constantemente, por lo 
que se ha actuado con la mayor discreción posible, por ello, no se han utilizado formatos de registro de la observación.

Con respecto a los sujetos estudiados, han sido cuidadosamente seleccionados según sugiere la estrategia empleada del estudio de casos: "el estudio de casos (...) se trata de una forma de elección de los sujetos u objetos para ser estudiados" (Colás, 1998, 257). De acuerdo con el carácter eminentemente cualitativo de la investigación y con la estrategia del estudio de casos, no es esencial para la investigación la amplitud de la muestra sino el análisis en profundidad del corpus. Los diferentes casos analizados se corresponden a alumnos con necesidades educativas especiales, derivadas principalmente de una patología visual. Se han estudiado un total de 17 alumnos con problemas visuales, que han sido agrupados en dos casos: uno, formado por alumnos con baja visión (11 sujetos) y, otro, compuesto por escritores invidentes ( 6 sujetos). La edad media de los sujetos estudiados es de 15.8 años (15 años y 8 meses), siendo la edad media de los alumnos con baja visión 15.6 y 16 la edad media de los invidentes. No obstante, se han seleccionado algunos sujetos con edades notablemente diferentes, tanto muy por debajo de la media (concretamente, 4 alumnos tienen una edad inferior a los 13 años) como por encima de ella (hay 6 alumnos con una edad superior a los 18 años). En efecto, la desviación típica de las edades de los sujetos con baja visión es 2.7 y la de los sujetos invidentes es 4 . La estrategia de ampliar el rango de edad de los sujetos investigados permite lograr uno de los propósitos de la investigación: analizar la influencia de la edad en el desarrollo de los procesos cognitivos de la expresión escrita. La edad cobra especial importancia si consideramos el "salto cognitivo" que experimentan los sujetos con deficiencia visual a la edad de 13-14 años, diferenciándose del patrón de desarrollo madurativo más uniforme o regular del sujeto normovidente, contradiciendo incluso los planteamientos piagetianos (Ochaíta, 1993). Asimismo, se contempla la prolongación, también peculiar, del desarrollo evolutivo de los sujetos con déficits visuales hasta la edad de 18 años (Ochaíta, 1993). Los alumnos con deficiencia visual se encuentran, generalmente, escolarizados en centros ordinarios, según establece la LOGSE en su propuesta de integración (aunque algunos se encuentran escolarizados en centros específicos), y reciben el apoyo material y personal necesario, a través de los centros educativos especializados para la atención a alumnos con necesidades educativas asociadas a la ceguera o a la baja visión (EAICDV).

\section{ANÁLISIS Y DISCUSIÓN DE RESULTADOS}

\section{1. Índice de complejidad numérica}

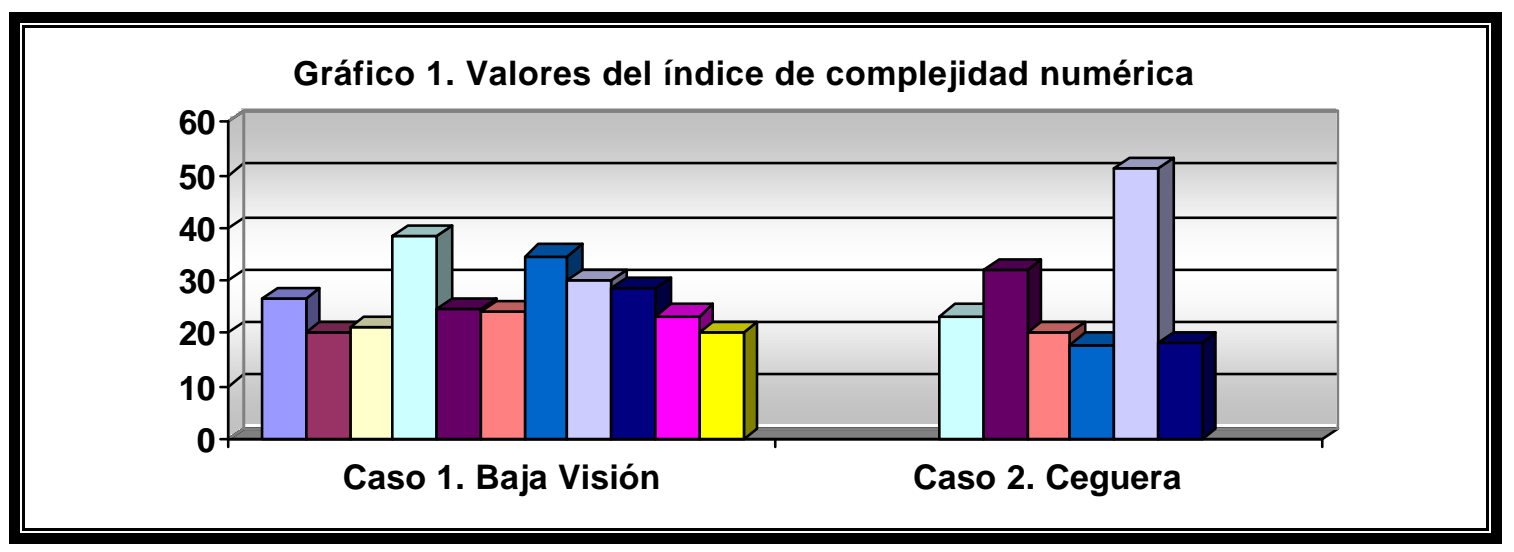


El valor medio de este índice para los textos construidos por alumnos con baja visión es 26.52, el rango es 18.5 y la desviación típica es 5.73. Los alumnos más jóvenes (sujetos 3, 7, 14 y 15) han obtenido valores inferiores a los de los alumnos mayores y de mayor nivel educativo (sujetos 2, 5, 10 y 11). En general, predomina un estructura proposicional binaria, que sólo en tres textos el índice alcanza la complejidad terciaria (sujetos 5 , 8 y 11 ).

De otra parte, el índice medio de los textos de alumnos invidentes es parecido al anterior: 27.13. Sin embargo, la dispersión entre los índices individuales es mayor (rango $=33.2$, desviación típica $=11.76$ ), lo cual indica que existen valores muy por encima de la media, mayores incluso que los valores más altos del caso anterior, como es el caso del sujeto 16 cuyo índice ha sido el más alto de todos los alumnos $(\mathrm{CP} 1=$ 51.2); pero también existen valores muy por debajo de la media, como es el caso de los sujetos más jóvenes (sujetos $12 \mathrm{CP} 1=20$ y $13 \mathrm{CP} 1=18$ ); y otro que recientemente se ha iniciado en la escritura Braille (sujeto $17 \mathrm{CP} 1=18.3$ ). En síntesis, la estructura de complejidad proposicional típica es la binaria, a excepción de dos sujetos cuyos textos han obtenido un índice de complejidad terciaria y de quinto grado, en el caso del alumno mayor.

Por tanto, la comparación individual de los índices de complejidad numérica denota una ligera superioridad de la mayoría de los alumnos con baja visión frente a los invidentes, salvo la excepción de los sujetos 9 y 16 (Cfr. Gráfico 1).

Tabla 1.

Distribución de textos según valores del índice CP1

\begin{tabular}{|c|c|c|c|}
\hline Indices & Salvador (1984) & Sujetos con Baja Visión & Sujetos Invidentes \\
\hline$<10$ & $1.9 \%$ (Infrecuente) & - & - \\
$11-15$ & $10.5 \%$ (Infrecuente) & - & - \\
$16-20$ & $29.7 \%$ (Poco frecuente) & $18.18 \%$ (Infrecuente) & $50 \%$ (Frecuente) \\
$21-25$ & $27.2 \%$ (Poco frecuente) & $36.36 \%$ (Poco frecuente) & $16.67 \%$ (Infrecuente) \\
$26-30$ & $19.2 \%$ (Infrecuente) & $27.27 \%$ (Poco frecuente) & - \\
$31-35$ & $6.2 \%$ (Infrecuente) & $9.09 \%$ (Infrecuente) & $16.67 \%$ (Infrecuente) \\
$36-40$ & $4.3 \%$ (Infrecuente) & $9.09 \%$ (Infrecuente) & - \\
$41-45$ & $0.6 \%$ (Infrecuente) & - & - \\
$46-50$ & $0.6 \%$ (Infrecuente) & - & - \\
$>50$ & - & - & $16.67 \%$ (Infrecuente) \\
\hline
\end{tabular}

Con respecto a la comparación de los sujetos de esta investigación con otros sin deficiencia visual, estudiados por otros autores (Salvador, 1984) existe cierta similitud entre los índices hallados a partir de los textos producidos por los alumnos invidentes y los hallados en otras investigaciones con alumnos sin deficiencia visual pero de menor edad (Cfr. Tabla 1), lo cual puede indicar un cierto retraso en la complejidad numérica de los textos creados por los invidentes. Por el contrario, los índices obtenidos de los textos de alumnos con baja visión muestran una complejidad superior con respecto a los alumnos sin deficiencia visual, como cabía esperar, dada la diferencia en la edad media de los sujetos de ambas investigaciones. 


\subsection{I Índice ponderado de complejidad}

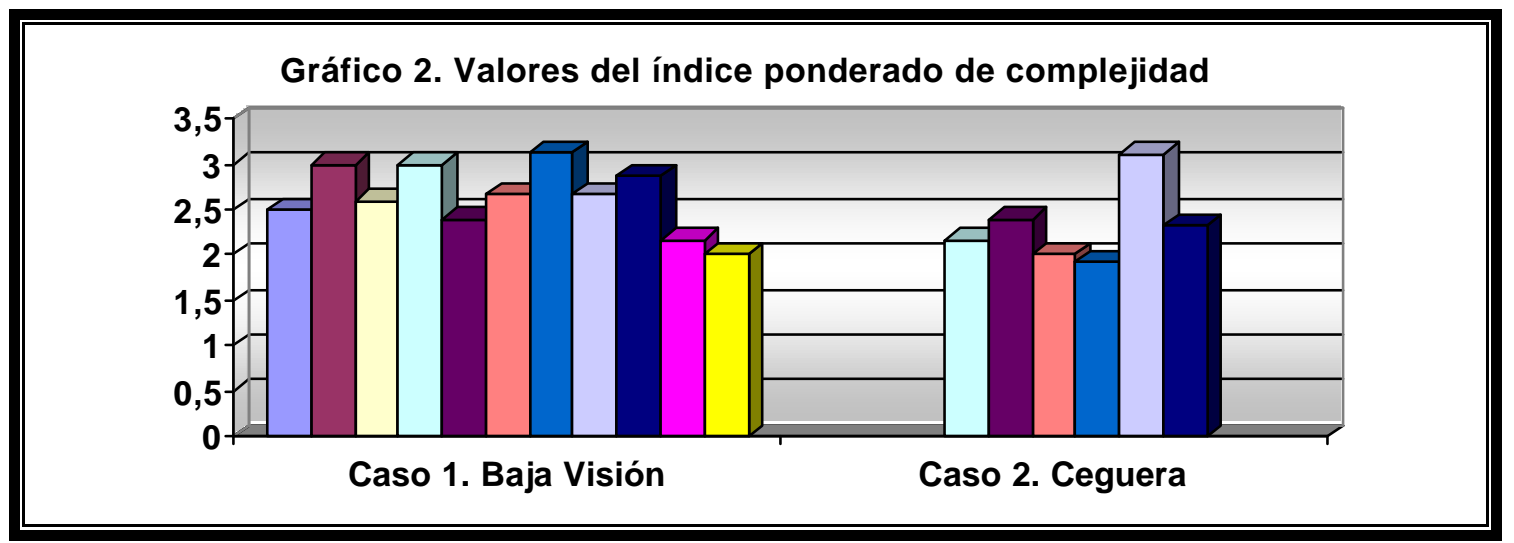

El índice total de todos los textos producidos por los alumnos con baja visión es 2.64, el rango es 1.13 y la desviación típica es pequeña (0.11), a pesar de que los alumnos más jóvenes (sujetos 14 y 15) han obtenido valores más bajos que el resto de sus compañeros (Cfr. Gráfico 2). Los valores obtenidos indican que la mayoría de los textos no alcanzan la subordinación de primer grado, siendo la media de los índices 2.64. Tan sólo algunos alumnos mayores (27.27\%) han obtenido el grado de subordinación de primer grado.

Los alumnos invidentes han obtenido un índice ponderado menor que los alumnos anteriores (2.32), el rango es 1.19 y la desviación típica es 0.15 . Los alumnos más jóvenes, igual que ocurría en el caso anterior, han obtenido valores menores (sujetos 12 y 13) que el resto, y los mayores valores mayores (sujeto 16). En este caso, predominan también las oraciones simples, independientes y coordinadas ante las subordinadas. En efecto, tan sólo un sujeto (9.09\%), el de mayor edad y nivel instructivo, ha alcanzado el grado cualitativo de subordinación de primer grado.

Tabla 2.

Distribución de textos según valores del índice CP2

\begin{tabular}{|c|c|c|c|}
\hline Indices & Salvador (1984) & Sujetos con baja visión & Sujetos invidentes \\
\hline$<1.5$ & $9.9 \%$ (Infrecuente) & - & $16.67 \%$ (Infrecuente) \\
$1.5-2$ & $23.5 \%$ (Poco frecuente) & - & $66.67 \%$ (Frecuente) \\
$2-2.5$ & $32 \%$ (Poco frecuente) & $27.27 \%$ (Poco frecuente) & - \\
$2.5-3$ & $22.8 \%$ (Poco frecuente) & $45.45 \%$ (Frecuente) & $16.67 \%$ (Infrecuente) \\
\hline$>3$ & $11.5 \%$ (Infrecuente) & $27.27 \%$ (Poco frecuente) & \multicolumn{2}{|c}{} \\
\hline
\end{tabular}

Por tanto, existen ciertas diferencias en cuanto a la complejidad de los textos entre alumnos con baja visión e invidentes, a favor de los primeros, según indica el índice ponderado de complejidad. Sin embargo, existe cierta similitud entre los índices hallados en los textos de alumnos sin deficiencia visual y los de alumnos invidentes, a pesar de la diferencia de edad, lo cual evidencia un cierto desfase en este aspecto. No ocurre lo mismo para el caso de los alumnos con baja visión, que han obtenido valores superiores, en general (Cfr. Tabla 2).

\subsection{I Índice de complejidad estructural de las oraciones}

En este índice, los valores más pequeños, es decir, que más se aproximan a "0", se corresponden con la complejidad mayor. 


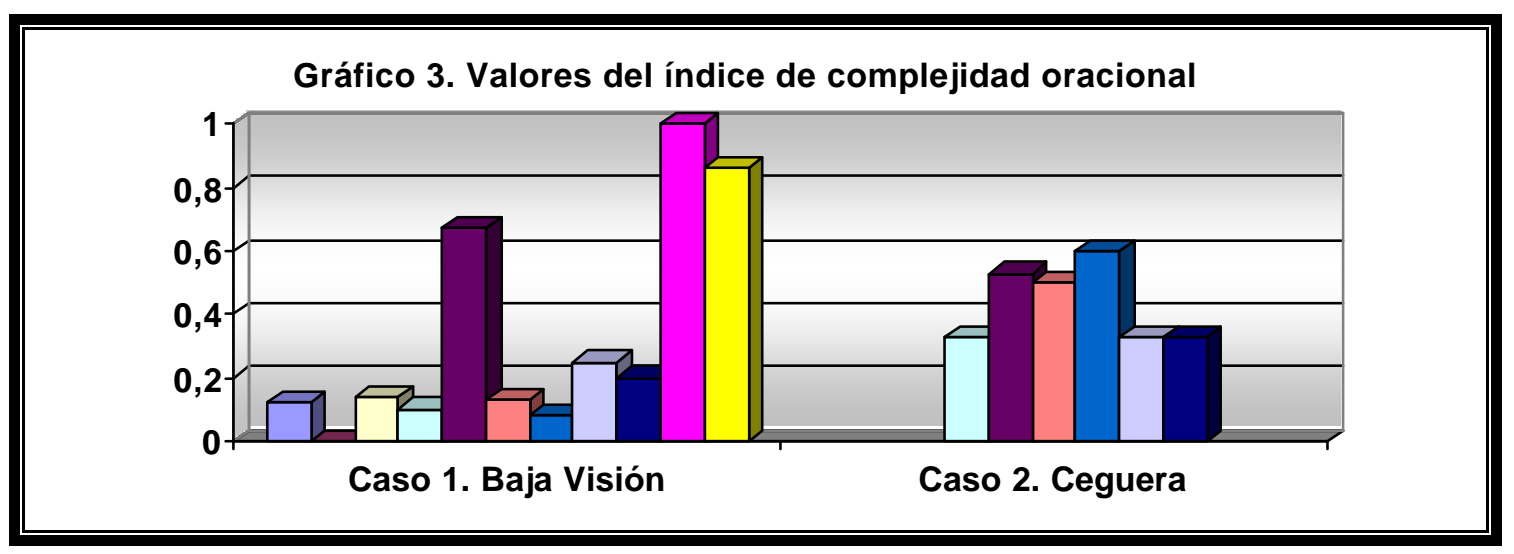

El valor medio del índice de complejidad estructural oracional para los alumnos con baja visión es 0.32 (Cfr. Gráfico 3) (rango = 1, con una desviación típica de 0.13 ), un valor bajo que indica una cantidad relativamente mayor de oraciones por subordinación que por coordinación y por yuxtaposición; conjuntamente, lo que denota un alto grado de complejidad sintáctica en los textos. Los textos que han obtenido valores más altos y, por tanto, son de menor complejidad sintáctica, corresponden a los alumnos más jóvenes, de Primaria (sujetos 14 y 15). Aún así, todos los textos han obtenido un valor inferior a 1, lo que denota un predominio de la modalidad de elaboración de oraciones por subordinación que por coordinación.

Ligeramente superiores son los índices de complejidad, obtenidos en los textos de alumnos invidentes (media $=0.44$, rango $=0.27$, desviación típica $=0.07$, lo cual indica una menor complejidad sintáctica, especialmente en los textos de los más jóvenes (sujetos 9, 12 y 13), como ocurría en el caso anterior (Cfr. Gráfico 3). Aunque también en todos los textos predomina la subordinación como modalidad de creación oracional.

Tabla 3.

Distribución de textos según valores del índice CP3

\begin{tabular}{|c|c|c|c|}
\hline Indices & Salvador (1984) & Sujetos con Baja Visión & Sujetos Invidentes \\
\hline 0 & $19 \%$ (Infrecuente) & $9.09 \%$ (Infrecuente) & - \\
\hline $0-0.5$ & $58 \%$ (Frecuente) & $63.64 \%$ (Bastante frecuen.) & $50 \%$ (Frecuente) \\
$0.5-1$ & & $27.27 \%$ (Poco frecuente) & $50 \%$ (Frecuente) \\
\hline $1-1.5$ & $23 \%$ (Poco frecuente) & - & - \\
\hline
\end{tabular}

Los textos de los alumnos con baja visión tienen una mayor complejidad (que se corresponden con los valores bajos del índice de complejidad estructural oracional: 0 - 0.5) que los de los alumnos invidentes (Cfr. Tabla 3). Ambos resultan más complejos que los textos de alumnos más jóvenes sin deficiencia visual (Salvador, 1984), a pesar de que en todos los casos el valor medio del índice es menor que 1, lo que denota un predominio de las estructuras por subordinación. Sin embargo, los textos de los alumnos sin deficiencia visual, en algunas ocasiones (23\%), contienen mayor cantidad de oraciones compuestas por coordinación o yuxtaposición, de menor complejidad sintáctica, aspecto que no se presenta en los textos de alumnos deficientes visuales. 
4.4. Iíndice de complejidad estructural de las proposiciones

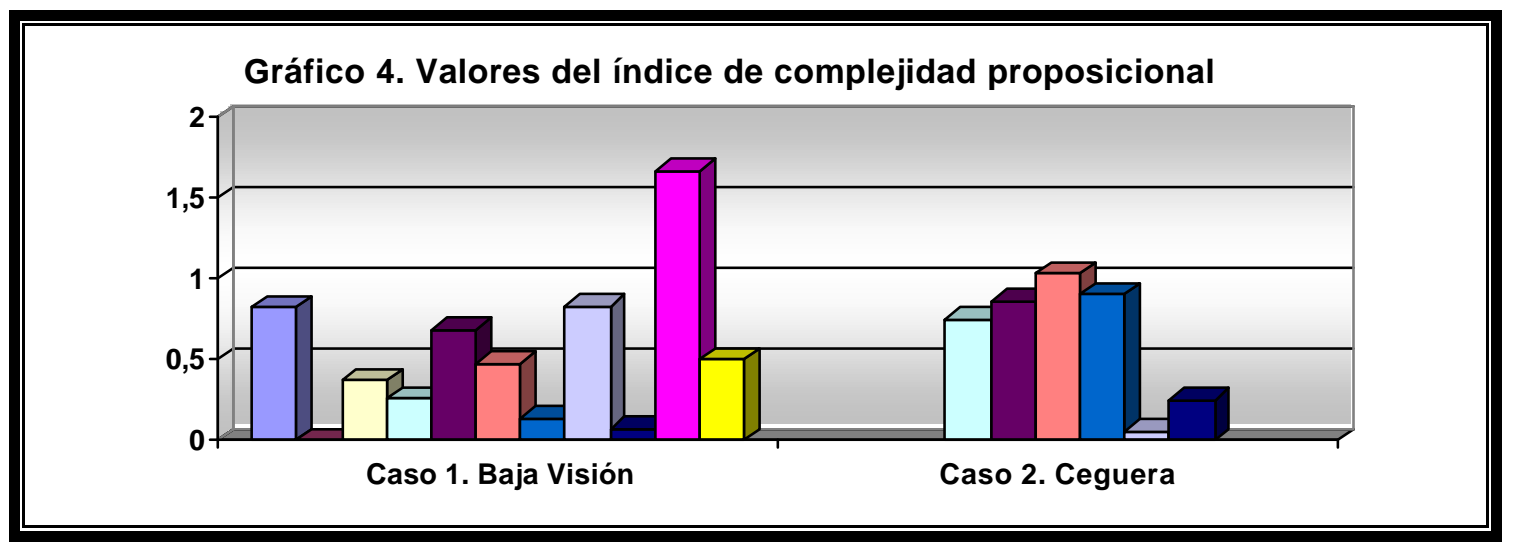

Los textos de alumnos con baja visión han obtenido un valor medio en el índice de complejidad proposicional de 0.53 (Cfr. Gráfico 4), con una dispersión de datos pequeña (rango $=0.83$, desviación típica $=0.18$ ). Los textos de los más jóvenes (sujetos 10, 14 y 15) son los que han obtenido, nuevamente, los valores más altos, es decir, una complejidad proposicional menor. Tan sólo uno de ellos (sujeto 14), el más joven, ha construido mayor cantidad de proposiciones coordinadas y yuxtapuestas que subordinadas. En el resto de los textos, predominan las proposiciones subordinadas.

Como en el caso de la construcción oracional, los alumnos invidentes han obtenido un índice algo superior (Cfr. Gráfico 4): 0.65 (rango $=0.97$, desviación típica $=0.26$ ), lo cual implica una complejidad ligeramente menor que los alumnos con cierto resto visual, especialmente los jóvenes (sujetos 9,12 y 13). A pesar de ello, tan sólo uno de ellos (sujeto 12), también el más joven como ocurriese en el caso de alumnos con baja visión, ha utilizado mayor número de proposiciones coordinadas y yuxtapuestas que subordinadas.

Tabla 4.

Distribución de textos según valores del índice CP4

\begin{tabular}{|l|c|c|c|}
\hline Índices & Salvador (1984) & Sujetos con Baja Visión & Sujetos Invidentes \\
\hline $0-0.5$ & & $54.54 \%$ (Frecuente) & $33.33 \%$ (Poco frecu.) \\
$0.5-1$ & $77 \%$ (Bastante frecuente) & $36.36 \%$ (Poco frecuente) & $50 \%$ (Frecuente) \\
$1-1.5$ & $23 \%$ & $9.09 \%$ (Infrecuente) & $16.67 \%$ (Infrecuente) \\
\hline
\end{tabular}

En síntesis, la complejidad proposicional resulta ser mayor en los textos de alumnos con baja visión que en los de alumnos invidentes (Cfr. Tabla 4). Además, los resultados obtenidos en los textos de alumnos invidentes se acercan a los obtenidos por Salvador (1984) (de 0 a 1 el $77 \%$ de alumnos sin deficiencia visual y el $83.33 \%$ de alumnos invidentes, y mayor que 1 el $23 \%$ de alumnos sin deficiencia visual y el $16.67 \%$ de alumnos invidentes), pese a que la edad de éstos es sensiblemente mayor que los de aquellos, variable relevante. Ello refleja un ligero desfase en la composición escrita de alumnos invidentes.

\section{CONCLUSIONES}

Parece evidente que la complejidad sintáctica encontrada en los textos producidos por alumnos con ceguera es menor que la encontrada en alumnos con baja visión, salvo algún texto excepcional. De otra parte, los textos producidos por alumnos con escasa visión son más complejos que los producidos por alumnos con visión normal de menor edad, según el contraste con los resultados extraídos con esta población por Salvador (1994), a diferencia de los textos producidos por alumnos sin visión, cuyos valores en los índices utilizados han resultado menores o semejantes que los obtenidos de los textos de alumnos 
con visión normal pero más jóvenes. Ello puede indicar un cierto desfase en la complejidad numérica, proposicional y oracional de los textos producidos por alumnos invidentes en relación a los producidos por alumnos con visión normal y con baja visión. En relación a estos dos grupos últimos, dado que no se disponen de suficientes estudios en nuestro contexto geográfico en cuanto a la progresión de la complejidad sintáctica ni de investigaciones sobre el análisis de la complejidad numérica y estructural de niños con edades análogas a las de esta investigación no se pueden establecer conclusiones.

\section{REFERENCIAS BIBLIOGRÁFICAS}

BARRAGA, Natalie, Textos reunidos de la doctora Barraga, Madrid, ONCE, 1990.

COLÁS, Pilar: Métodos y técnicas cualitativas de investigación en psicopedagogía, en BUENDíA, Leonor y otras (Eds.), Métodos de Investigación en psicopedagogía, Madrid, McGraw-Hill, 1998.

OCHAíTA, Esperanza, Ceguera y desarrollo psicológico, en ROSA, Alberto y OCHAÍTA, ESPERANZA (Comps.), Psicología de la Ceguera, Madrid, Alianza Editorial, 1993.

RODRíGUeZ, Antonio y MARTínez, Francisco, Atención educativa a ciegos y deficientes visuales, Granada, Arial Ediciones, 2002.

RODRíGUEZ, Antonio, Análisis de la expresión escrita en alumnos con n.e.e. vinculadas a la deficiencia visual, Tesis Doctoral, Universidad de Granada, 2002.

RODRíGUEZ, Antonio, La expresión escrita en alumnos con deficiencia visual, Granada, Arial Ediciones, 2003.

RODRíGUEZ, Antonio, ¿Cómo leen niños con ceguera y baja visión?, Málaga, Aljibe, 2005.

SALVADOR, Francisco, "El análisis de textos infantiles. Aportaciones teórico-metodológicas y didácticas", Enseñanza, 4-5, 1986, pp 221-236.

SALVADOR, Francisco, El aprendizaje lingüístico y sus dificultades, Granada, Grupo Editorial Universitario, 1999.

SALVADOR, Francisco, Cómo prevenir las dificultades en la expresión escrita, Málaga, Aljibe, 2000.

SIMON, Jean, La langue ecrite de l'enfant, Paris, Presses Universitaries de France, 1973. 


\title{
Contactar
}

Revista lberoamericana de Educación

\author{
Principal OEI
}

